\title{
Apigenin Ameliorates Insulin Resistance and Lipid Accumulation by Endoplasmic Reticulum Stress and SREBP-1c/SREBP-2 Pathway in Palmitate-Induced HepG2 Cells and High-Fat Diet-Fed Mice ${ }^{\mathbb{3}}$
}

\author{
Liling Wu, Tingdong Guo, Ranxi Deng, Lusheng Liu, and Yongxiong Yu \\ College of Animal Science and Technology, Southwest University, Chongqing, China (L.W., L.L., Y.Y.) and Department of \\ Pharmacy, Nanchong Central Hospital, The Second Clinical Medical College (L.W., T.G.), Department of Clinical Medicine (R.D.), \\ North Sichuan Medical College, Nanchong, Sichuan, China
}

Received October 6, 2021; accepted January 21, 2021

\begin{abstract}
Insulin resistance (IR) is the common basis of diabetes and cardiovascular diseases, and its development is closely associated with lipid metabolism disorder. Flavonoids have definite chemical defense effects, including anti-inflammatory effects, anticancer effects, and antimutation effects. However, the function and mechanism of apigenin (AP, a kind of flavonoid) in IR are still unclear. In our study, intracellular fat accumulation model cells and high-fat diet (HFD)-fed model mice were established using palmitate (PA) and HFD. Mechanistically, we first demonstrated that AP could notably downregulate sterol regulatory element-binding protein 1c (SREBP-1c), sterol regulatory element-binding protein 2 (SREBP-2), fatty acid synthase, stearyl-CoA desaturase 1, and 3-hydroxy-3-methyl-glutarylCoA reductase in PA-induced hyperlipidemic cells and mice. Functionally, we verified that AP could markedly reduce lipid accumulation in PA-induced hyperlipidemic cells and decrease the body weight, visceral fat weight, IR, and lipid accumulation in HFD-induced hyperlipidemic mice. Besides, we showed that PA could significantly downregulate endoplasmic reticulum stress
\end{abstract}

(ERS)-related proteins and inhibit ERS. Furthermore, we proved that AP could reduce blood lipids by inhibiting ERS in PAinduced hyperlipidemic cells. Meanwhile, 4-phenyl butyric acid (also called ERS alleviator), like AP, could significantly reduce blood lipids and alleviate IR in HFD-fed model mice. Therefore, we concluded that AP could substantially improve the disorder of lipid metabolism, and its mechanism might be related to the decrease of SREBP-1c, SREBP-2, and downstream genes, the inhibition of ERS, and the reduction of blood lipids and IR.

\section{SIGNIFICANCE STATEMENT}

Apigenin, a nontoxic and naturally sourced flavonoid, has antihyperlipidemic properties in mice and hepatocyte. This study highlights a new mechanism of apigenin and proposes that these hypolipidemic effects are associated with the mitigation of endoplasmic reticulum stress and insulin resistance in dietinduced obesity. This study might provide translational insight into the prevention and treatment of apigenin in hyperlipidemiarelated diseases.

\section{Introduction}

As the most crucial metabolic and biotransformative organ in the body, the liver plays a pivotal role in lipid metabolism, especially in maintaining the balance of lipid metabolism (Gröger et al., 2018). When the balance of lipid metabolism is broken by various reasons, the liver is often the first to be affected, causing the accumulation of lipids in liver cells,

The authors declare no financial conflict of interest. This work was supported by the National Key program of Ministry of Science and Technology of China [Grant 2018YFD0502000].

https://doi.org/10.1124/jpet.120.000162.

S This article has supplemental material available at jpet.aspetjournals.org steatosis, and even tumorigenesis ( $\mathrm{Ni}$ and Wang, 2016). At present, much evidence has manifested that the occurrence and development of fatty liver are in connection with various factors, such as dyslipidemia, insulin resistance (IR), oxidative stress, lipid peroxidation, and apoptosis, which are also present the characteristics of cascade reaction (Awad et al., 2016). Therefore, it is of great significance for the prevention and treatment of fatty liver and other hyperlipidemia-related diseases to improve liver lipid metabolism, enhance the antioxidant capacity of liver tissues, and facilitate liver metabolism of lipids, sugars, and other substances through a drug intervention.

Apigenin (AP), a nontoxic and mutation-resistant flavonoid, is frequently found in fruits and vegetables such as grapefruit

ABBREVIATIONS: Akt, protein kinase B; AP, apigenin; ATF6, activating transcriptional factor 6; CHOP, C/EBP homologous protein; elF2A, eukaryotic initiation factor-2A; ER, endoplasmic reticulum; ERS, endoplasmic reticulum stress; FAS, fatty acid synthase; GRP78, glucose-regulated protein 78; HDL-C, high-density lipoprotein cholesterol; HFD, high-fat diet; HMGCOR, 3-hydroxy-3-methyl-glutaryl-CoA reductase; IGTT, intravenous glucose tolerance test; IR, insulin resistance; IRE1, inositol-requiring enzyme 1; IRS1, insulin receptor substrate 1; LDL-C, low-density lipoprotein cholesterol; LO, lovastatin; OGT, oral glucose tolerance test; p-, phosphorylated; PA, palmitate; 4-PBA, 4-phenyl butyric acid; PERK, protein kinase RNA-like ER kinase; SCD-1, stearyl-CoA desaturase 1; SREBP-1c, sterol regulatory element binding protein 1c; SREBP-2, sterol regulatory element binding protein 2; TC, total cholesterol; TG, triglyceride; Tudca, tauroursodeoxycholate; Tun, tunicamycin. 
and celery (Nabavi et al., 2018). In recent years, numerous studies have discovered that AP has high chemical defense effects, including antimutation, anti-inflammatory, antioxidation, and anticancer effects (Salehi et al., 2019). According to the recent reports, AP could inhibit the differentiation of 3T3-L1 preadipocytes (Guo et al., 2016), alleviate the glucose metabolic disorders in mice fed a high-fat diet for 4 weeks at the age of 20 weeks (Jung et al., 2016), and prevent secondary obesity after weight loss by improving gut microbes (Chilloux and Dumas, 2017). Thus, we concluded that AP could alleviate obesity induced by a high-fat diet and obesity-related metabolic diseases. Besides, pharmacological experiments showed that AP had hypoglycemic, anti-inflammatory, and antioxidant effects on diabetic mice (El Barky et al., 2019). Another study by Li et al. (2017) revealed that AP could improve blood pressure and arteriosclerosis in hypertensive animal models. However, AP's treatment mechanism for hyperlipidemia and its effects on lipid and insulin resistance have not been clearly elucidated.

Endoplasmic reticulum stress (ERS) refers to the aggregation state of misfolded and unfolded proteins in the lumen under the condition of homeostasis imbalance in the endoplasmic reticulum (ER) (Iurlaro and Muñoz-Pinedo, 2016). The physiologic and pathologic factors of ERS include excessive intracellular protein synthesis, metabolic disorders, inflammatory responses, and calcium homeostasis ( $\mathrm{Li}$ et al., 2017). To improve ERS, the ER can activate several signaling pathways to restore cellular functions, including enhancing protein folding, halting the translation of most proteins, and accelerating protein degradation (Lebeaupin et al., 2018). At present, the relationship between ERS and the process of metabolic diseases has become an active area of research. A growing number of studies have shown that ERS is closely associated with IR (Suzuki et al., 2017; Ramos-Lopez et al., 2018; Maiers and Malhi, 2019). However, it is not clear whether the effects of AP on lipid and IR can be achieved by ERS.

The purpose of this study was to investigate the effects of AP on serum lipids and IR in hyperlipidemia mice. Simultaneously, in vitro experiments further confirmed the regulatory impact of AP on sterol regulatory element-binding protein 1c (SREBP-1c) and sterol regulatory element-binding protein 2 (SREBP-2) pathways and ERS-related proteins. Moreover, we also revealed the effects of AP and ERS on hyperlipidemia. Therefore, our study might provide a theoretical and experimental basis for the prevention and treatment of AP in fatty liver and other hyperlipidemia-related diseases.

\section{Materials and Methods}

Cell Culture and Treatment. HepG2 cells (cat. no. A019) were acquired from American Type Culture Collection. Cells were grown in Dulbecco's modified Eagle's medium (cat. no. 5796; Sigma) with $10 \%$ fetal calf serum (Gibco) at $37^{\circ} \mathrm{C}$ with $5 \% \mathrm{CO} 2$. For treatment, HepG2 cells were treated with $0,0.1,1,5,10,25,50,100$, and $200 \mu \mathrm{M}$ AP. Besides, the intracellular fat accumulation model cells were established using $250 \mu \mathrm{M}$ palmitate (PA; cat. no. P5586; SigmaAldrich), and the model cells were administered $25 \mu \mathrm{M}$ AP, $10 \mu \mathrm{M}$ lovastatin (LO, a positive control), $10 \mu \mathrm{M}$ tunicamycin (Tun, an ERS agonist, cat. no. 150028; Sigma), or $5 \mathrm{mM}$ tauroursodeoxycholate (Tudca, an ERS inhibitor), respectively.

Establishment and Treatment of Hyperlipidemia Model Mice. Six-week-old healthy male C57BL/6J mice (weighing between
20 and $21 \mathrm{~g}$ ) were obtained from Shanghai Experimental Animal Center. Mice were first fed adaptively for 1 week. Feeding conditions included adequate food and water, comfortable temperature $24 \pm 1^{\circ} \mathrm{C}$, and 12-hour alternate light/dark cycles. The animal experimental protocols in this study were approved by the Animal Experimentation Ethics Committee of Southwest University. Mice were fed a conventional diet (Medicine, Jiangsu, China, cat. no. MD12062) and a highfat diet (HFD; cat. no. MD12032; Medicine). The high-fat diet group mice were treated with $\mathrm{AP}(10 \mathrm{mg} / \mathrm{kg}$ ig) and LO (10 mg/kg ig) or 4phenyl butyric acid (4-PBA; $200 \mathrm{mg} / \mathrm{kg}$ i.p.) for 12 weeks. In the experiment processes, $300 \mathrm{~g}$ diets per week were added into each small cage ( 2 to 3 mice per cage) to ensure sufficient food; then the food intake was examined per cage weekly, and the accumulative food intake of per cage was calculated for 12 weeks. Meanwhile, the body weight of each group of mice was examined once a week for 12 weeks. The mice were anesthetized, blood was collected, and the liver tissues and visceral fat (the fat in the groin and omentum were taken as visceral fat weight) were obtained and weighed at 12 weeks.

Cell Counting Kit-8 Assay. HepG2 cells during logarithmic growth were inoculated in 96 -well plates with $3 \times 10^{3}$ cells per well. AP was added to the HepG2 cells with concentrations of $0,0.1,1,5,10$, $25,50,100$, and $200 \mu \mathrm{M}$, respectively. After 48 hours of conventional culture, $15 \mu \mathrm{l}$ Cell Counting Kit-8 solution (Sigma-Aldrich) was added to each well and incubated for 3 hours. The absorbance optical density of each well was detected at $450 \mathrm{~nm}$ with a microplate reader.

Western Blot Assay. Radio immunoprecipitation assay lysis buffer, including proteases inhibitor, was applied to extract the total proteins of $50 \mathrm{mg}$ of liver tissues or the treated HepG2 cells. After centrifugation at $4^{\circ} \mathrm{C}$, the supernatant was taken. After the protein concentration was determined by the bicinchoninic acid method, the total protein $(40 \mu \mathrm{g})$ in each group was separated by $12 \%$ SDS-PAGE electrophoresis. Subsequently, the proteins were transferred into polyvinylidene fluoride membranes through the electrophoretic transfer method. After sealing, the membranes were incubated with primary antibodies at $4^{\circ} \mathrm{C}$ overnight. The membranes were then treated with horseradish peroxidase-labeled goat anti-rabbit or antimouse secondary antibodies (1:5000) at room temperature for 2 hours. After the reaction of enhanced chemiluminescence reagent (Bio-Rad) for 5 minutes, the results were obtained through developing imaging in a dark room. Primary antibodies contained SREBP-1c (ab28481; Abcam), SREBP-2 (ab30682; Abcam), phosphorylated protein kinase RNA-like ER kinase (p-PERK; sc-32577; Santa Cruz), protein kinase B (AKT; 55230-1; Proteintech), p-AKT Ser473 (28731-1; Proteintech), insulin receptor substrate 1 (IRS1; 2382; Cell Signaling Technology), p-IRS1 Y612 (12628; Abnova), glucose-regulated protein 78 (GRP78; ab32618; Abcam), phosphorylated eukaryotic initiation factor-2A (eIF2 $\alpha$; ab227593; Abcam), C/EBP homologous protein (CHOP; ab10444; Abcam), activating transcriptional factor 6 (ATF6; sc16659; Santa Cruz), inositol-requiring enzyme 1 (IRE1; sc-390960; Santa Cruz), and Tubulin (Cell Signaling Technology).

Immunofluorescence Assay. Based on the experimental groups, cells were inoculated in the cell slides of the six-well plate and cultured overnight. After rinsing with PBS, the cells were fixed with $4 \%$ neutral paraformaldehyde for 15 minutes and treated with $0.25 \%$ Trixon- 100 for 5 minutes. After washing, $5 \%$ bovine serum albumin was applied to seal cells at $37^{\circ} \mathrm{C}$ for 1 hour, and anti-SREBP-1c ( $5 \mu \mathrm{g} / \mathrm{ml}$, ab28481; Abcam) and anti-SREBP-2 $(1 \mu \mathrm{g} / \mathrm{ml}, \mathrm{ab30682}$; Abcam) were used to treat cells overnight at $4^{\circ} \mathrm{C}$. The next day, the cells were hatched with the second antibody for 2 hours. DAPI was added to the cell slides, and the results were observed under a fluorescence microscope.

Quantitative Real-Time Polymerase Chain Reaction Assay. The liver tissues and the treated HepG2 cells were applied to extract the total RNAs through the usage of TRIzol reagent (Invitrogen). NanoDro 2000c (Thermo Scientific) was applied to examine the quality of RNAs. Next, $2 \mu \mathrm{g}$ RNAs, as a template, were used to generate cDNA via the utilization of PrimeScript RT Reagent (Takara, Dalian, China). Gene levels were confirmed using the SYBR Premix Taq kit (Takara) on an ABI 7300 Real-Time system (Applied Biosystems). 
Oil Red Staining. The liver tissues of the mice were quickly taken out, frozen, and sliced ( 5 to $6 \mu \mathrm{m}$ ). The slices were fixed using $4 \%$ formaldehyde, washed using $100 \%$ propylene glycol, and dyed with Oil Red O g overnight in darkness. After the glycerine gelatin was used to seal the slices, the results were observed and photographed under the microscope.

Biochemical Index Detection. All mice were fasting for 12 hours and intraperitoneally injected with $2 \%$ sodium pentobarbital $(0.3 \mathrm{ml} / 100 \mathrm{~g})$. Blood was taken from the femoral artery, and serum was separated. A biochemical analyzer was applied to analyze the levels of triglyceride (TG), total cholesterol (TC), high-density lipoprotein cholesterol (HDL-C), and low-density lipoprotein cholesterol (LDL-C). Serum leptin was detected by ELISA kit, and HOMA-IR(homeostasis model assessment of insulin resistance) was calculated by formula FBG(fasting blood glucose) $\times$ FINS(fasting insulin)/22.5 .

Glucose Tolerance and Pancreatic Insulin Tolerance. After the mice were deprived of food for 10 hours, they were given $2 \mathrm{~g} / \mathrm{kg}$ glucose by gavage or $0.1 \mathrm{U} / \mathrm{kg}$ insulin by intraperitoneal injection. Blood glucose was collected at $0,30,60$, and 120 minutes, respectively. The vital signs of the mice were normal during the blood collection. The levels of blood sugar were measured.

Statistical Analysis. All experimental data are shown as means \pm S.D. and counted using the GraphPad Prism (version 7.0). The results were estimated through one-way ANOVA analysis or Student's $t$ test. $P<0.05$ represented statistical significance.

\section{Results}

AP Notably Downregulated the Levels of SREBP-1c, SREBP-2, and Their Downstream Genes in PA-Induced Intracellular Fat Accumulation Model HepG2 Cells. To determine AP's possible mechanism and its impact on the fat accumulation in hepatoma cells, the intracellular fat accumulation cell model was built using PA. Firstly, the role of AP on the viability of HepG2 cells was determined through the Cell Counting Kit-8 assay. The results showed that AP could prominently refrain the viability of HepG2 cells in the doseeffect relationship, and $25 \mu \mathrm{M}$ AP was applied to subsequent experiments based on the results. Meanwhile, we discovered that the cell viability was gradually decreased with the extension of AP processing time in HepG2 cells, especially 24 and 48 hours, and a 24 -hour processing time was chosen in subsequent experiments $(P<0.05, P<0.01, P<0.001$; Supplemental Fig. 1, A and B). Secondly, the fat accumulation model cells were administered $25 \mu \mathrm{M}$ AP and positive control (LO). The Western blotting results revealed that the levels of SREBP-1c and SREBP-2 were significantly increased in the PA-induced model group relative to the control group. Then AP treatment could prominently reduce SREBP-1c and SREBP-2 levels in PA-induced model cells $(P<0.01, P<$ 0.001; Fig. 1A). Simultaneously, the results of the immunofluorescence assay exhibited the same expression trends for SREBP-1c and SREBP-2 expressions as the Western blotting results $(P<0.05, P<0.01, P<0.001$; Fig. 1B). Thirdly, our results further revealed that the levels of fatty acid synthase (FAS), stearyl-CoA desaturase 1 (SCD-1), and 3-hydroxy-3methyl-glutaryl-CoA reductase (HMGCOR) were significantly increased in the PA-induced model group versus the control group. AP treatment markedly reduced FAS, SCD-1, and HMGCOR expressions induced by PA in HepG2 cells $(P<$ $0.05, P<0.01, P<0.001$; Fig. $1, \mathrm{C}$ and $\mathrm{D})$. Moreover, the results of oil red staining showed that intracellular fat accumulation mode cells had a large number of lipid droplets, w AP-treated cells had significant improvements in the number and size of lipid droplets, indicating that $\mathrm{AP}$ had a good effect on lipid reduction $(P<0.05, P<0.01, P<0.001$; Fig. 1E). Consistent with this, AP significantly inhibited the intracellular TG accumulation in PA-induced HepG2 cells $(P<0.05, P<0.001$; Fig. $1 \mathrm{~F})$. To sum up, our results demonstrated that the AP could inhibit SREBP-1c and SREBP-2 signaling pathways and reduce lipid accumulation in PA-induced HepG2 cells.

AP Markedly Reduced the Body Weight, Visceral Fat Weight, and Lipid Levels of HFD-Induced Hyperlipidemia Model Mice. Next, we estimated the influence of AP on the body weight, visceral fat weight, and blood lipids in HFDfed mice. To investigate the roles of AP, the HFD-fed mice were administered $\mathrm{AP}$ or $\mathrm{LO}$, and mice in each group were also examined (Fig. 2A). In the experiment process, the food intake was examined once a week for 12 weeks, and changes of serum leptin were detected at the end of 12 weeks. The results indicated that the accumulative food intake was obviously elevated in HFD-fed mice compared with ND-fed mice, whereas the elevation of food consumption mediated by HFD could be moderately decreased by AP or LO medication in mice, suggesting that the food intake affected the bodyweight of mice to some extent (Supplemental Fig. 2A). Besides, we proved that the level of leptin serum was dramatically elevated in HFD-fed mice compared with that in the NC mice, whereas the elevation of leptin serum level could also be markedly attenuated by AP or LO in HFD-fed mice, indicating that AP or LO observably reduced leptin resistance in HFDfed mice $(P<0.05, P<0.01$; Supplemental Fig. 2B). Subsequently, the body weight of mice was monitored once a week for 12 weeks, and the data revealed that the body weight of mice was notably elevated in the HFD model group versus the control group, whereas this elevation mediated by HFD could be notably reversed by AP administration in mice, and the change had a time-effect relationship $(P<0.001$; Fig. 2B). At the end of the experiment, the liver weight and visceral fat weight were also examined. The liver weight and visceral fat weight were prominently increased in the HFD model group compared with the control group. Simultaneously, the increasing effects induced by HFD could be observably attenuated by $\operatorname{AP}(P<0.01, P<0.001$; Fig. $2, \mathrm{C}$ and $\mathrm{D})$. Besides, our results proved that TG, TC, and LDL-C were obviously elevated, and HDL-C was notably reduced in the HFD model group relative to the control group. These changes could be remarkably weakened by AP administration in mice $(P<0.05$, $P<0.01, P<0.001$; Fig. 2, E-H). Hence, we showed that AP could exhibit remarkable roles in reducing the body weight, visceral fat weight, and lipid levels in HFD-fed model mice.

AP Effectively Alleviated IR and Lipid Accumulation and Reduced SREBP-1c and SREBP-2 Expressions in HFD-Fed Model Mice. Subsequently, more experiments were performed to deeply study the biologic functions of AP in HFD-fed model mice. Firstly, through intraperitoneal glucose tolerance test (IPGTT) and oral glucose tolerance test (OGTT), the fasting blood glucose of mice was measured by a glucose meter. As exhibited in Fig. 3, A and B, the concentrations of blood glucose were notably elevated in the HFD model group versus the control group. The elevation of blood glucose in HFD-fed model mice could be significantly attenuated by AP administration $(P<0.001)$. Secondly, oil red staining was applied to assess the impact of AP on the lipid accumulation in HFD-fed model mice. The results revealed 
that HFD could result in a notable increase for the lipid accumulation in mice, whereas this increase could be notably reversed by AP administration $(P<0.001$; Fig. 3C). Finally, we showed that HFD feeding could cause upregulation of SREBP-1c and SREBP-2 expressions in hepatic tissues of mice, whereas AP could then weaken upregulation in HFD-fed model mice $(P<0.001$; Fig. 3D). Furthermore, HFD feeding could cause downregulation of IR markers p-Akt and p-IRS1. At the same time, AP could weaken downregulation in hepatic tissues of HFD-fed model mice $(P<0.001$; Fig. 3E). Moreover, we demonstrated that HFD feeding could also result in a significant reduction in p-Akt and p-IRS1 expressions, whereas only LO could moderately elevate the level of p-Akt mediated by HFD in the gastrocnemius muscle and inguinal fat of mice. So, we revealed that liver is a targeting tissue of AP for protection against IR; other insulin-sensitive tissues (gastrocnemius tissue and inguinal fat) were not the effective targets of AP for protection against IR $(P<0.05, P<0.01, P<$ 0.001; Supplemental Fig. 3). Overall, these data confirmed that AP had significant inhibitory effects on IR and lipid
A

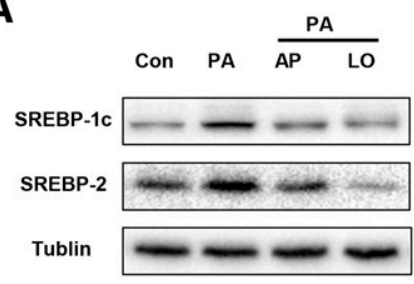

SREBP-1C

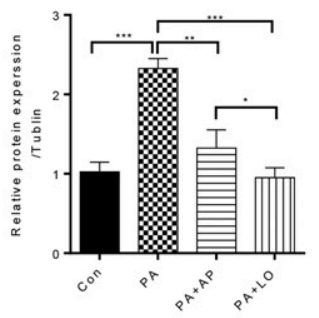

SREBP-2

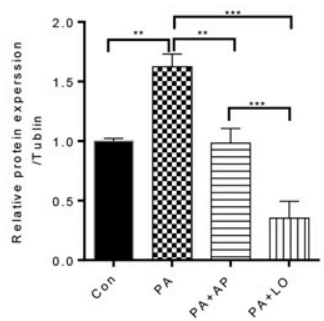

B

Con

PA

PA+AP

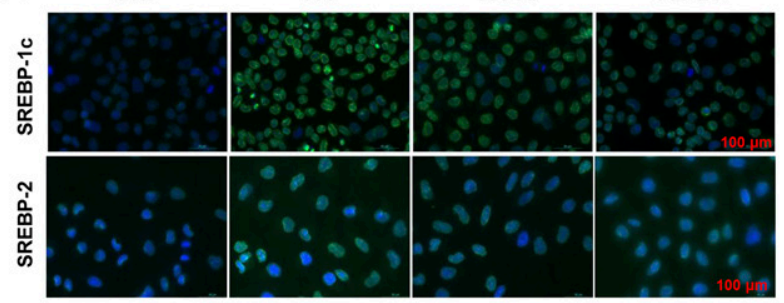

C
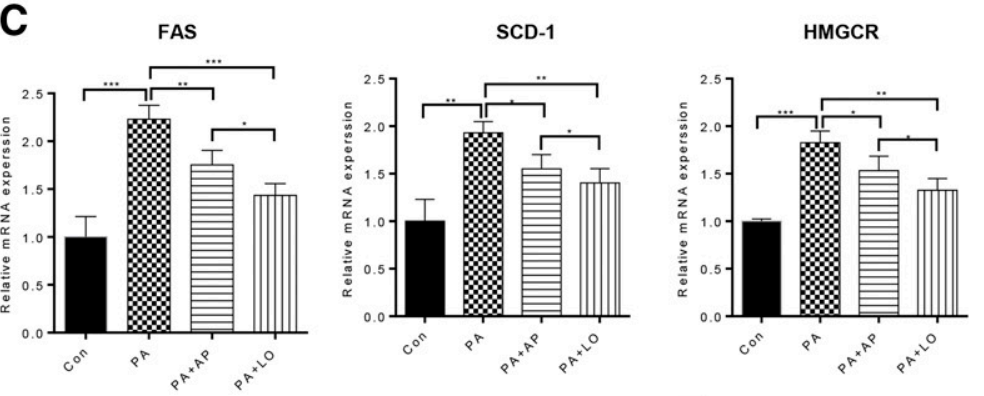

D
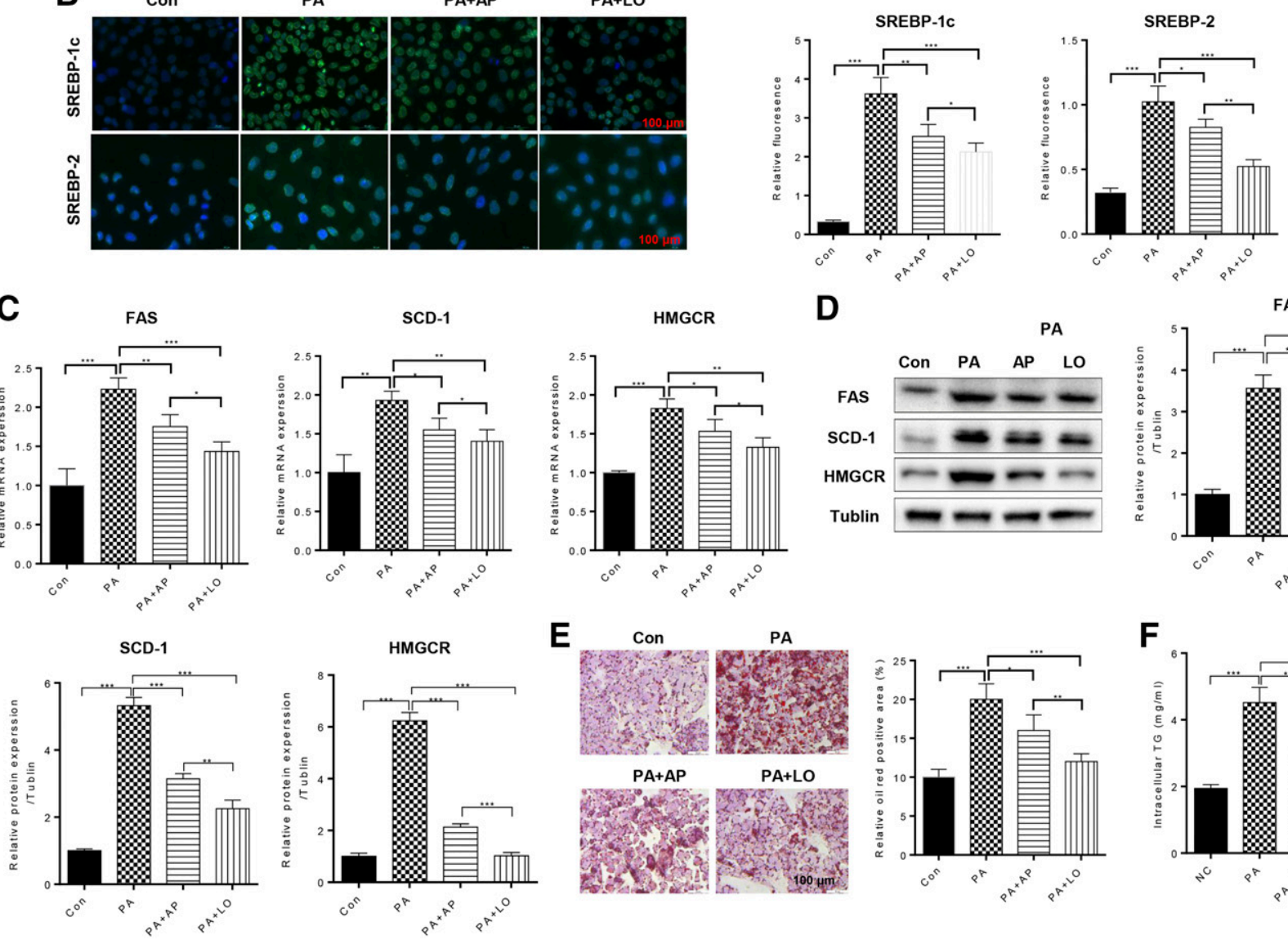

$\mathbf{E}$
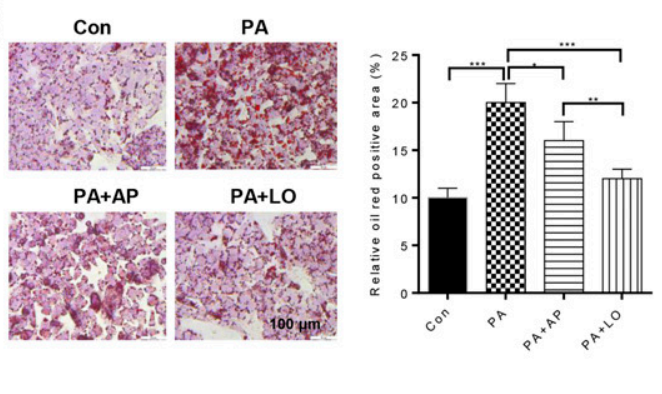

$\mathbf{F}$

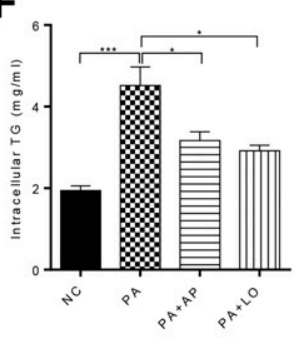

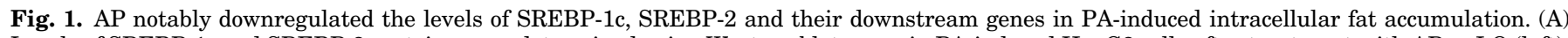

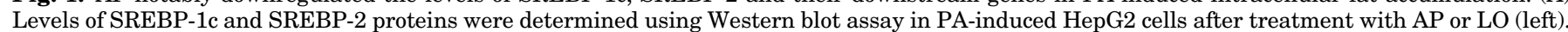

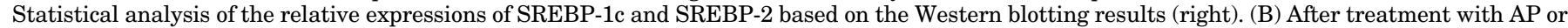

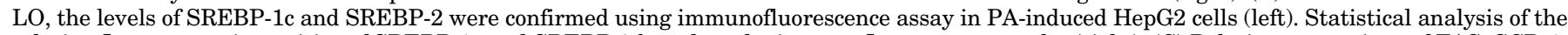

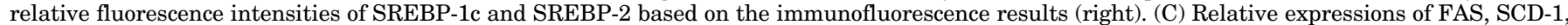

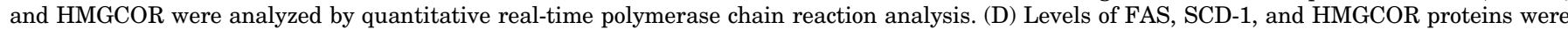

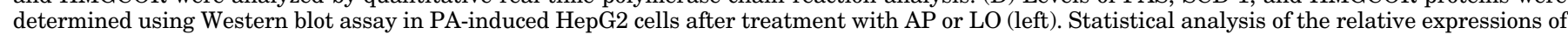

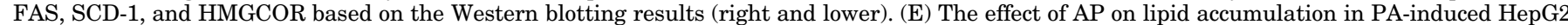

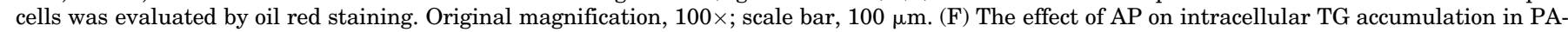

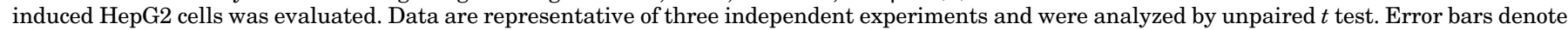
S.D. $* P<0.05 ; * * P<0.01 ; * * * P<0.001$. Con (control) 

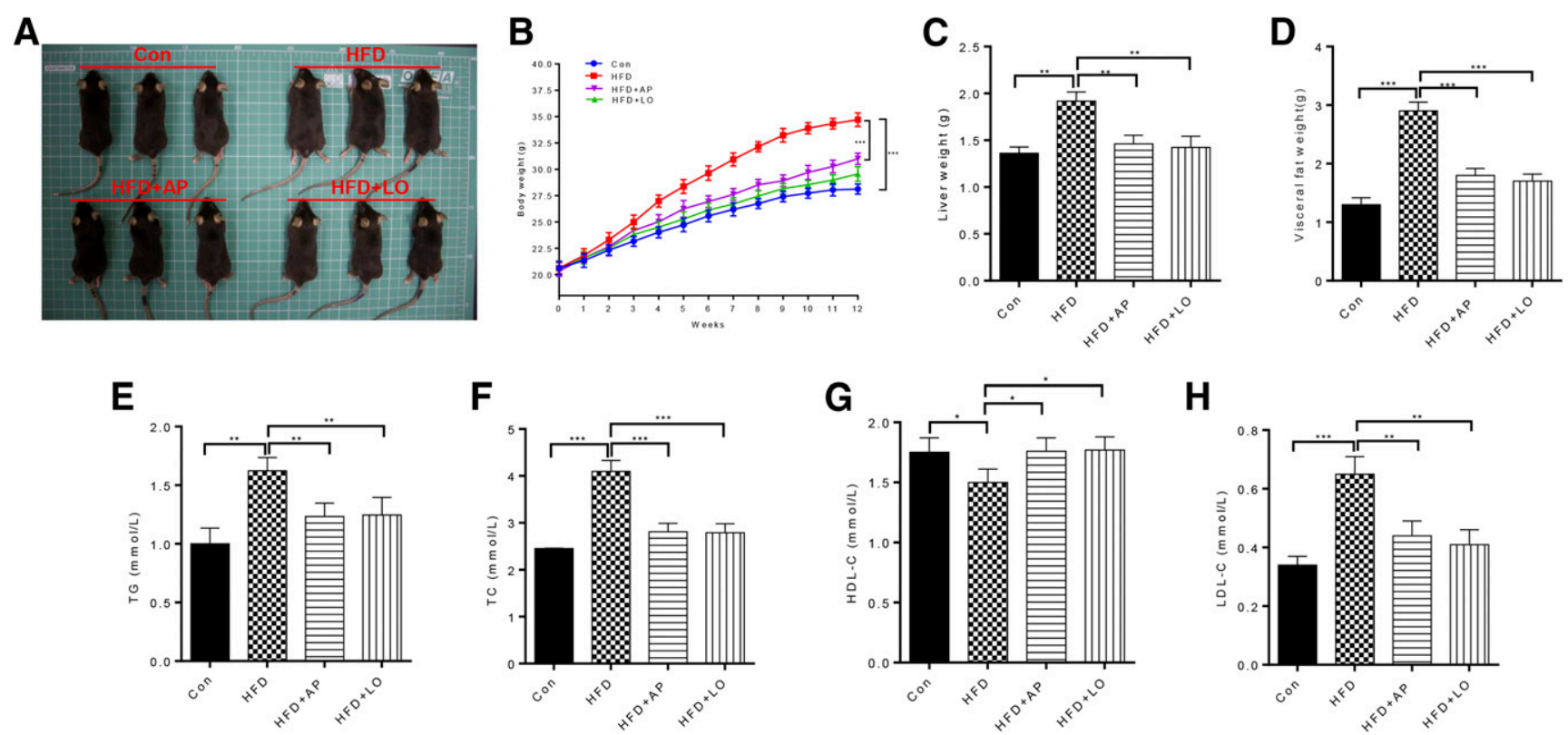

Fig. 2. AP markedly reduced the body weight, visceral fat weight, and lipid levels of HFD-induced hyperlipidemia model mice. (A) The HFD-fed mice model was established using HFD, and the mice were also treated with AP or LO. There were eight mice in each group. (B) The body weight was detected once a week for 12 weeks. The liver weight (C) and visceral fat weight (D) were also measured at the end of the experiment. TG (E), TC (F), HDL-C (G), and LDL-C $(\mathrm{H})$ were analyzed using a biochemical analyzer. Data are representative of three independent experiments and were analyzed by unpaired $t$ test. Error bars denote S.D. $* P<0.05 ; * * P<0.01 ; * * * P<0.001$. Con (control)

accumulation and downregulated SREBP-1c and SREBP-2 expressions in HFD-fed model mice.

AP Markedly Decreased Blood Lipids by Suppressing ERS in PA-Induced Intracellular Fat Accumulation Model HepG2 Cells. More importantly, we further investigated whether ERS plays a crucial role in the hypolipidemic process of AP. Firstly, ERS agonist (Tun) or AP was applied to treat the PA-induced HepG2 cells. Our data from the Western blot assay showed that the levels of ERS-related proteins (p-PERK, GRP78, p-eIF2 $\alpha$, and CHOP) were significantly increased in PA-induced HepG2 cells versus HepG2 cells (control), whereas the increase mediated by PA could be reversed by AP administration in HepG2 cells. Meanwhile, we revealed that Tun could significantly induce the upregulation of ERS-related proteins in PA-induced HepG2 cells $(P<$ $0.05, P<0.01, P<0.001 ;$ Fig. 4A). Secondly, the data showed that Tun prominently upregulated SREBP-1c and SREBP-2 expression. Simultaneously, the upregulation induced by Tun could be weakened by AP as well as Tudca (ERS inhibitor) in HepG2 cells $(P<0.001 ;$ Fig. 4B). Thirdly, our results showed that Tun dramatically increased ERS-related proteins, whereas the increases induced by Tun could be attenuated by AP or Tudca in HepG2 cells $(P<0.05, P<0.001$; Fig. 4 C). Moreover, our results discovered that Tun memorably promoted lipid accumulation, whereas the promotion induced by Tun could be reversed by AP or Tudca in HepG2 cells $(P<$ 0.001; Fig. 4D). In general, our results pointed out that the remarkable inhibitory effect of AP on lipid accumulation could be achieved by regulating ERS.

AP Prominently Reduced Blood Lipids in HFD-Fed Model Mice. Likewise, we further explored the roles of AP and ERS on blood lipids in HFD-fed model mice. HFD-fed model mice were administered with AP. Our Western blot assay data showed that the levels of ERS-related proteins (p-PERK, GRP78, p-eIF2 $\alpha$, and CHOP) were obviously upregulated in
HFD-fed model mice compared with the control mice, whereas AP could reverse the upregulation mediated by HFD in mice. So, we proved AP could prominently suppress ERS in HFD-fed model mice $(P<0.05, P<0.01, P<0.001$; Fig. 5A). Meanwhile, we also identified the effects of AP on the HOMA-IR, ATF6, and IRE1 expression in HFD-fed model mice. The data showed that the level of HOMA-IR was significantly increased in HFD-fed mice compared with that in NC mice, which could also be dramatically weakened by $\operatorname{AP}(P<0.01, P<0.001$; Supplemental Fig. 4A), whereas ATF6 and IRE1 expressions were not affected by AP in HFD-fed mice $(P<0.01, P<0.001$; Supplemental Fig. 4, B and C). Furthermore, HFD-fed model mice were administered with AP or 4-phenyl butyric acid (4-PBA, ERS alleviator). The Western blot assay data showed that AP had significant inhibitory effects on SREBP-1c and SREBP-2 expression in HFD-fed model mice $(P<0.05, P<$ 0.001 ; Fig. 5B). Meanwhile, we revealed that the levels of p-PERK, GRP78, p-eIF2 $\alpha$, and CHOP could be downregulated not only by AP but also by 4-PBA in HFD-fed model mice $(P<$ $0.05, P<0.001$; Fig. 5C). Furthermore, the oil red staining results showed that the lipid accumulation in liver tissues could be suppressed by AP in HFD-fed model mice $(P<0.05$, $P<0.001$; Fig. 5D). On the whole, we verified that AP could reduce SREBP-1c and SREBP-2 expressions, ERS, and lipid accumulation in HFD-fed model mice, suggesting the crucial inhibitory effects of AP and ERS in lipid accumulation of HFDfed model mice.

AP Markedly Reduced Blood Lipids and Alleviated IR in HFD-Fed Model Mice. Moreover, we further affirmed that ERS, like AP, could reduce blood lipid and IR in HFD-fed model mice. HFD-fed model mice were administered AP or 4PBA (Fig. 6A). First of all, our data displayed that 4-PBA, like $\mathrm{AP}$, could memorably reduce the body weight (Fig. $6 \mathrm{~B}$ ), liver weight (Fig. 6C), and visceral fat weight (Fig. 6D) of HFD-fed model mice $(P<0.01, P<0.001)$. Simultaneously, our results 

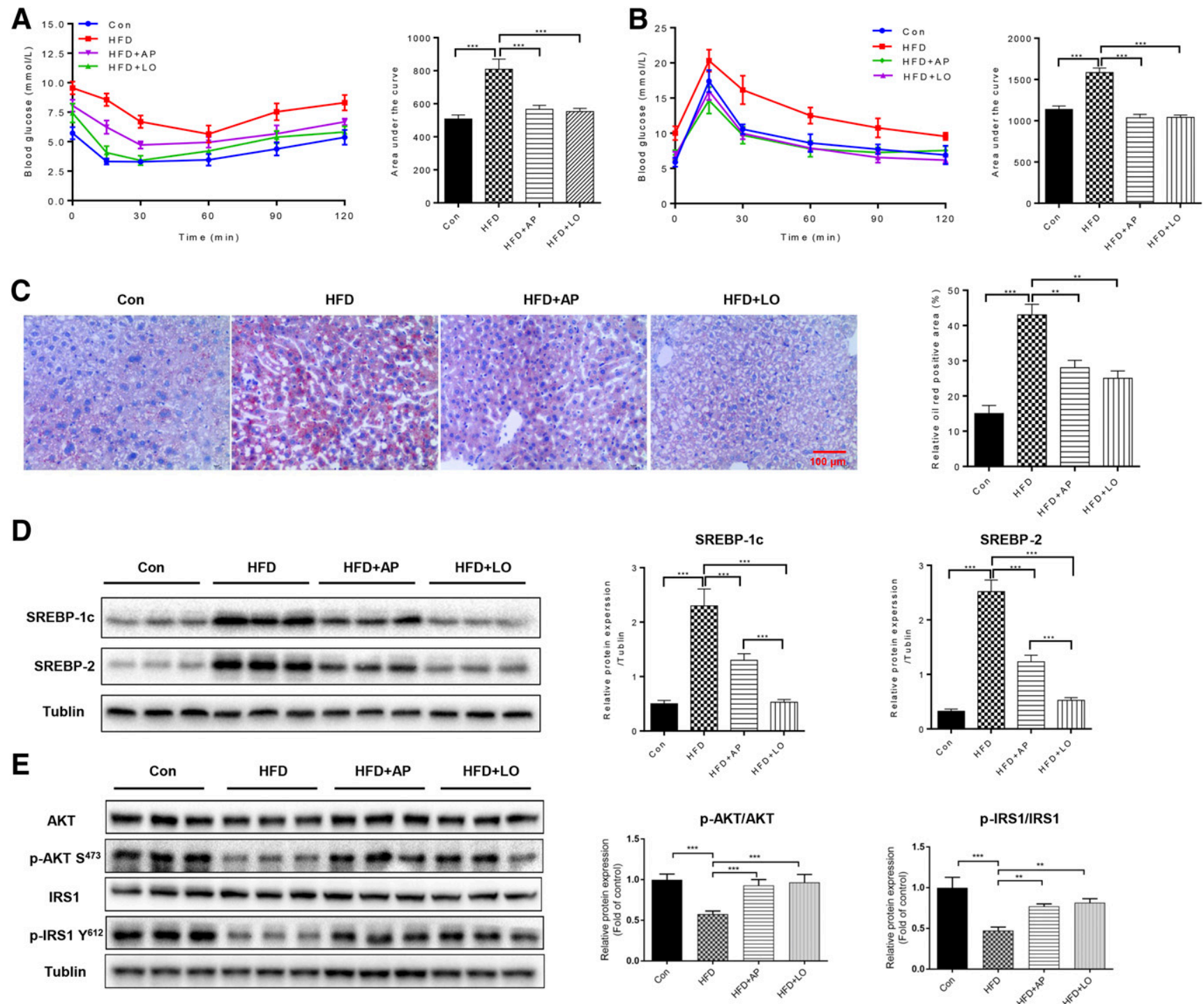

Fig. 3. AP effectively alleviated IR and lipid accumulation and reduced SREBP-1c and SREBP-2 expressions in HFD-fed model mice. (A and B) Blood glucose concentration was examined through IGTT and OGTT in HFD-fed model mice after treatment with AP or LO. (C) The lipid accumulation was assessed using oil red staining in HFD-fed model mice after AP or LO treatment. Original magnification, $100 \times$; scale bar, $100 \mu \mathrm{m}$ (left). Statistical analysis of the relative oil red positive area (right). (D) Levels of SREBP-1c and SREBP-2 proteins were determined using Western blot assay in HFD-fed model mice treated with AP or LO (left). Statistical analysis of the relative expressions of SREBP-1c and SREBP-2 based on the Western blotting results (right). (E) Levels of p-Akt and p-IRS1 were determined using Western blot assay in HFD-fed model mice treated with AP or LO (left). Statistical analysis of the relative expressions of p-Akt and p-IRS1 based on the Western blotting results (right). Data are representative of three independent experiments and were analyzed by unpaired $t$ test. Error bars denote S.D. $* P<0.05 ; * * P<0.01 ; * * P<0.001$. Con (control)

revealed that 4-PBA was similar to $\mathrm{AP}$ in that it obviously reduced TG, TC, and LDL-C and notably elevated HDL-C in HFD-fed model mice $(P<0.05, P<0.01, P<0.001$; Fig. 6 , E-H). Besides, our data showed that AP could notably reduce the concentrations of blood glucose in HFD-fed model mice via insulin tolerance test and OGTT ( $P<0.001$; Fig. 6, I and J). These results demonstrated that $\mathrm{AP}$ and 4-PBA could reduce blood lipids and alleviate IR in HFD-fed model mice.

\section{Discussion}

HepG2 cells, as a human liver carcinoma cell line, basically retain the biologic characteristics of normal liver cells and are enriched with high-affinity insulin receptors on the surface (Chen et al., 2019). HepG2 cells are currently widely applied to study fatty liver (Chen et al., 2018). PA is one of the most common saturated fats in the diet (Malik et al., 2019). The culture medium containing PA can induce the HepG2 cells to produce IR and can also better simulate the nutritional obesity status of the human liver (Mo et al., 2019). Besides, previous research has also demonstrated that PA could induce lipid accumulation and prevent lipid decomposition in hepatocytes (Zhao et al., 2017). Currently, the intracellular fat accumulation model induced by PA has become a classic model of fatty liver. In our study, intracellular fat accumulation cells were built using PA in HepG2 cells, and the underlying mechanism was explored. The SREBPs family includes three main subtypes: SREBP-1a, SREBP-1c, and SREBP-2 (DeBoseBoyd and Ye, 2018). SREBP-1c is a crucial transcriptional regulator of fatty acids and TC, which can regulate the key 
A

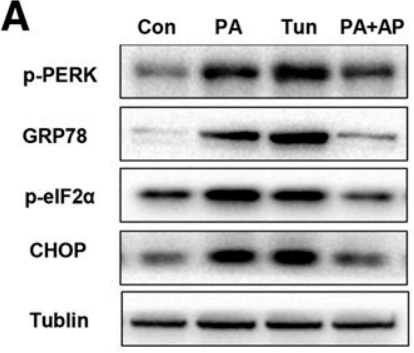

B

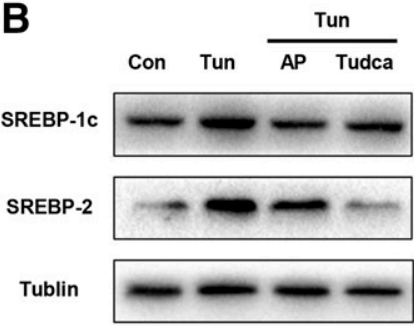

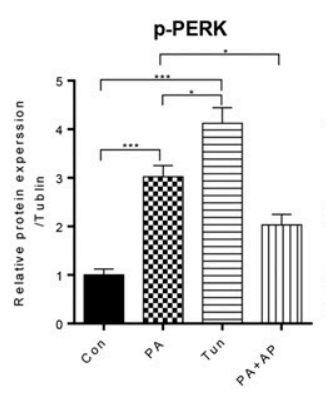

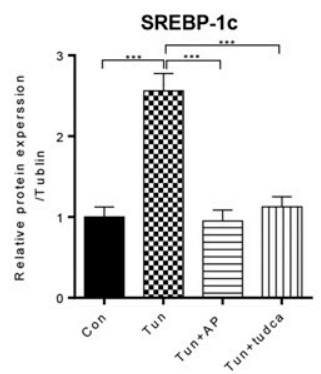

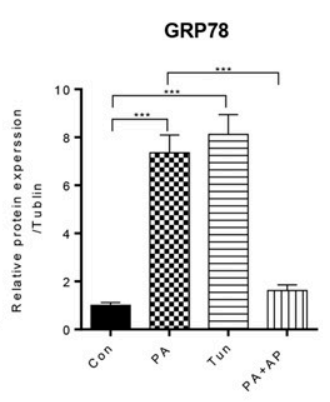

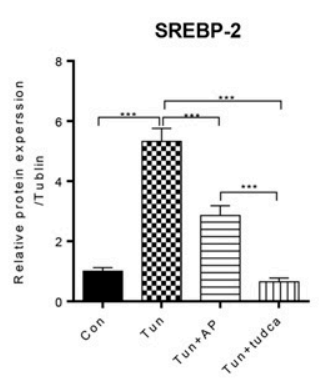

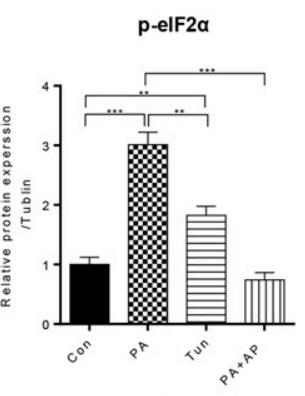

C

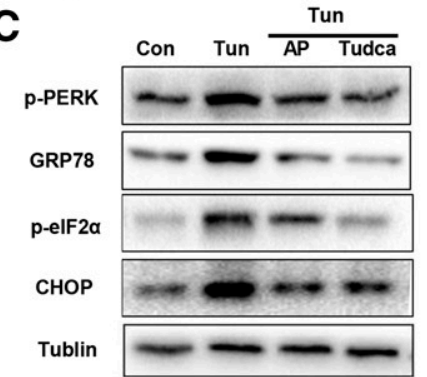

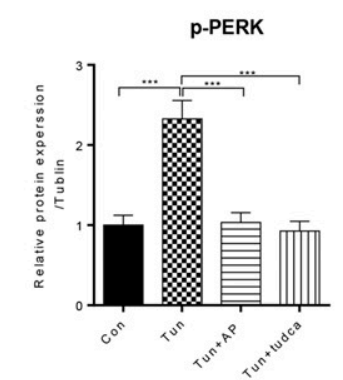

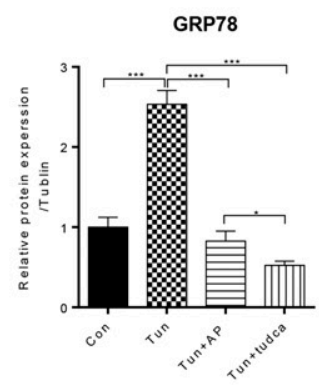

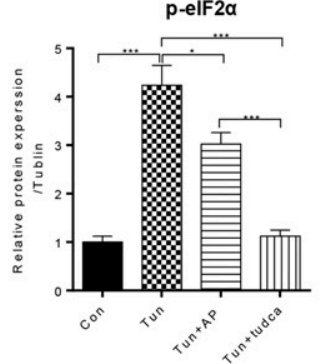

Tun+Tudca

D

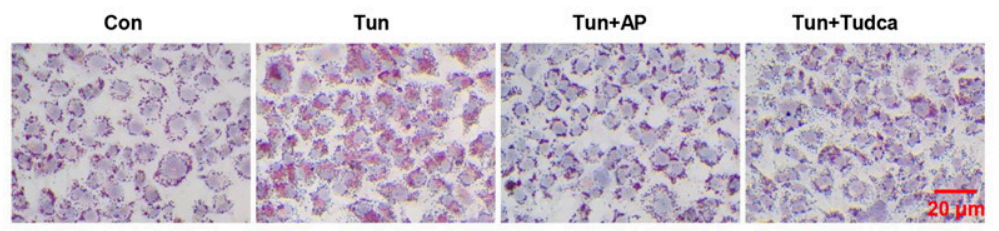

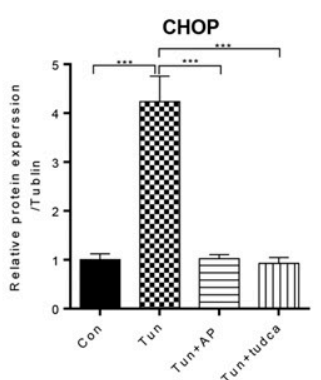

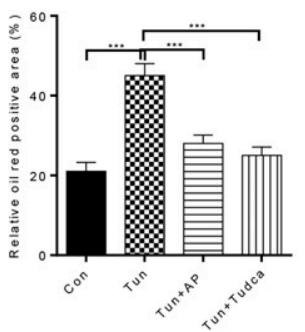

Fig. 4. AP markedly decreased blood lipids by suppressing ERS in PA-induced intracellular fat accumulation model HepG2 cells. (A) The PA-induced HepG2 cells were treated with ERS agonist (Tun) or AP, and the levels of ERS-related proteins (p-PERK, GRP78, p-eIF2 $\alpha$, and CHOP) were confirmed by Western blotting analysis (left). Statistical analysis of the levels of ERS-related proteins (p-PERK, GRP78, p-eIF2 $\alpha$, and CHOP) based on Western blot assay results (right). (B) Western blot assay was also used to determine the influences of AP and ERS on SREBP-1c and SREBP-2 expressions (left). Statistical analysis of the influences of AP and ERS on SREBP-1c and SREBP-2 expressions based on Western blot assay results (right). (C) The regulatory effects of AP and ERS on ERS-related proteins were also analyzed by Western blot assay (left). Statistical analysis of the regulatory effects of AP and ERS on ERS-related proteins based on Western blot assay results (right). (D) The effects of AP and ERS on lipid accumulation were assessed by oil red staining in PA-induced HepG2 cells. Original magnification, $400 \times$; scale bar, $20 \mu \mathrm{m}$ (left). Statistical analysis of the relative oil red positive area (right). Data are representative of three independent experiments and were analyzed by unpaired $t$ test. Error bars denote S.D. $* P<0.05 ; * * P<0.01$; $* * * P<0.001$. Con (control)

enzymes in lipid synthesis, including FAS and SCD-1 (Hernandez-Rodas et al., 2017; Kim et al., 2018). SREBP-2 is a crucial transcription factor in lipid metabolism, which is involved in regulating fatty acid and cholesterol synthesis (Gatineau and Yiannikouris, 2018). Among them, HMGCOR is the critical downstream gene of SREBP-2 (Khaleel et al., 2018). In this study, we showed that the levels of SREBP-1c, SREBP-2, FAS, SCD-1, and HMGCOR were notably upregulated in PA-induced HepG2 cells. Meanwhile, the lipid accumulation was also significantly enhanced in PA-induced HepG2 cells.

AP is a naturally occurring flavonoid with a wide range of physiologic and pharmacological effects, such as antitumor, antioxidative, anti-inflammatory, free radical scavenging, radiation damage protection, and differentiation regulation effects (Ren et al., 2018). Compared with other flavonoids, such as quercetin and kaempferol, AP has the characteristics of low toxicity and no mutagenicity (Madunić et al., 2018). In recent years, AP has been applied in the studies of various diseases, including cancers (Nabavi et al., 2015; Vrhovac Madunic et al., 2018), autoimmune disease (Kasiri et al., 2018), cardiomyopathy (Li et al., 2017), and neurogenic disease (Nabavi et al., 2018; Pang et al., 2018). Previous studies have also confirmed that AP has a significant regulatory effect on lipid metabolism. For example, AP could alleviate nonalcoholic 


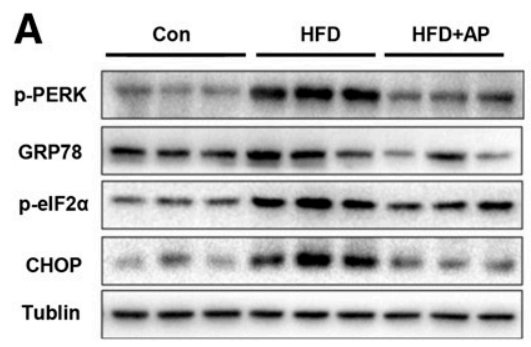

B
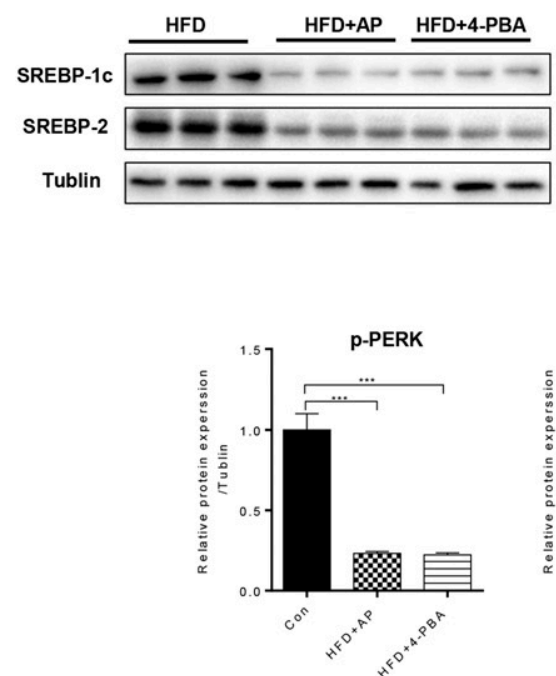

D

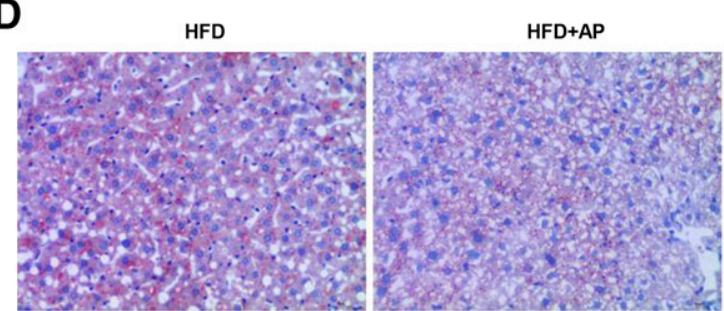

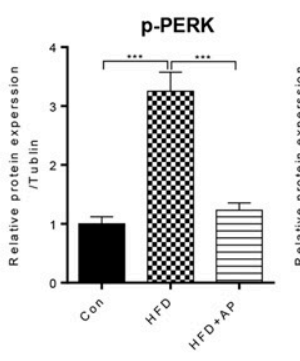
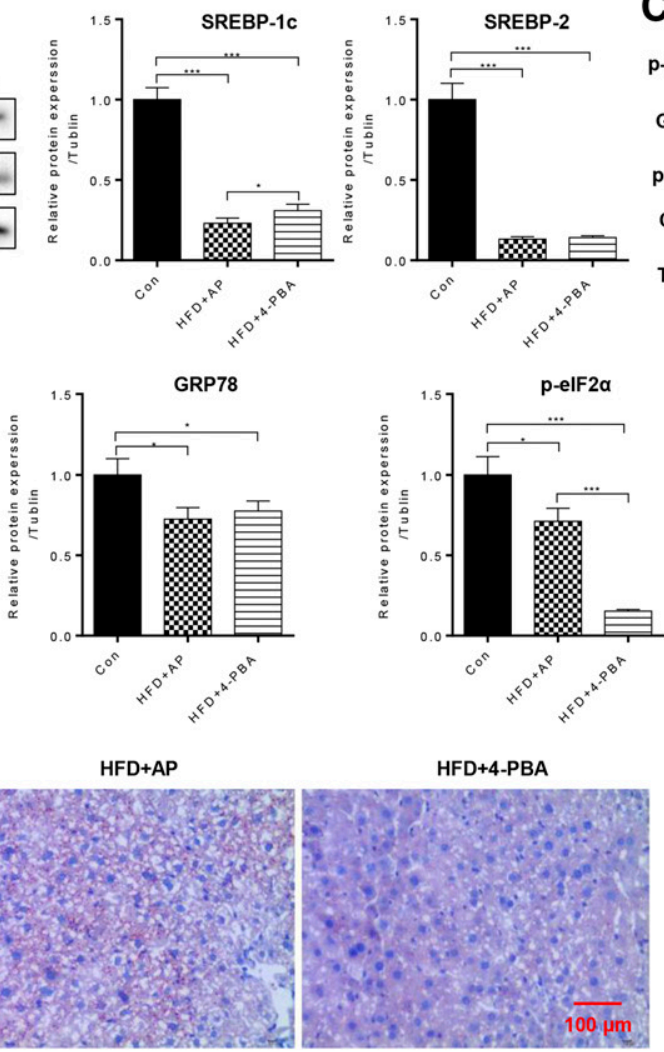

C
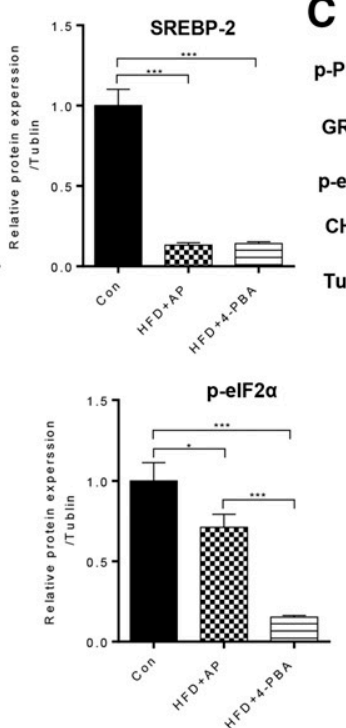
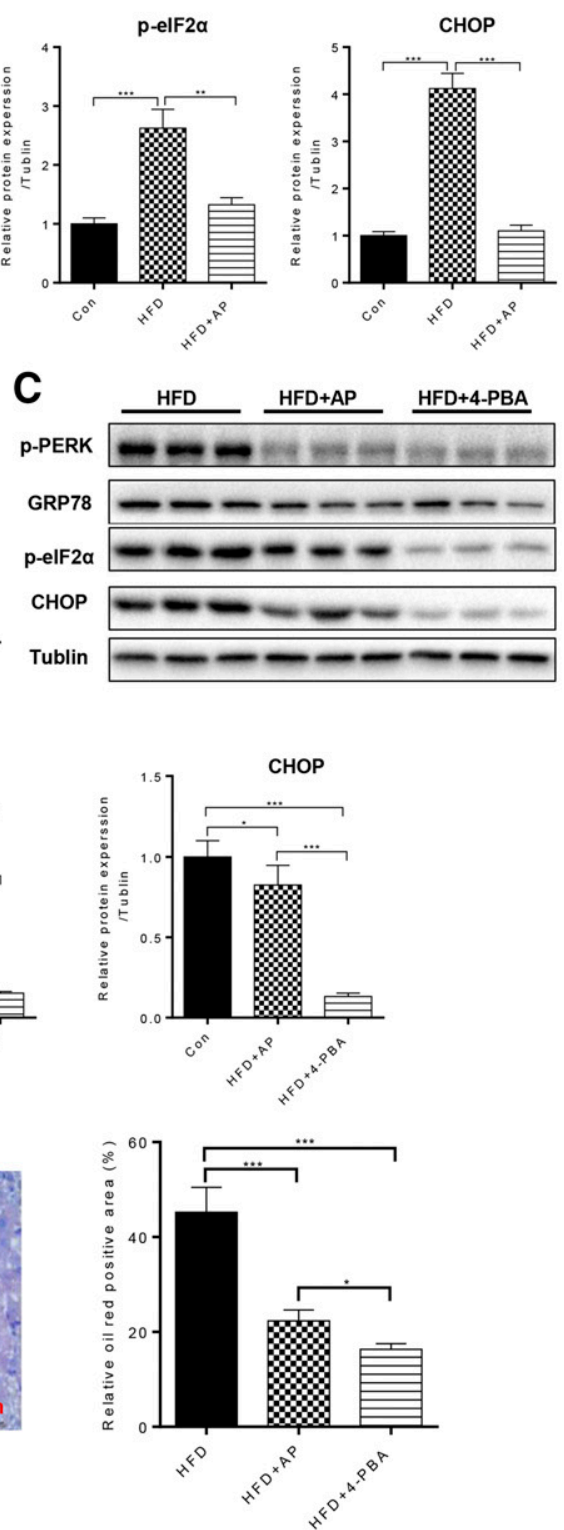

Fig. 5. AP prominently reduced blood lipids in HFD-fed model mice by ERS. (A) The levels of ERS-related proteins were determined by applying Western blot assay in the HFD-fed model mice after administration with AP (left). Statistical analysis of the levels of ERS-related proteins based on Western blot assay results (right). (B) After treatment with AP or 4-PBA, SREBP-1c and SREBP-2 expressions were monitored by Western blot assay in HFD-fed model mice (left). Statistical analysis of 4-PBA, SREBP-1c, and SREBP-2 expressions based on Western blot assay results (right). (C) Western blot assay was also applied to measure the impacts of AP and 4-PBA on ERS-related proteins levels (left). Statistical analysis was conducted to assess the impacts of AP and 4-PBA on ERS-related proteins levels based on Western blot assay results (right). (D) The influences of AP and 4-PBA on lipid accumulation were confirmed by oil red staining in HFD-fed model mice (left). Statistical analysis of the relative oil red positive area (right). Original magnification, $100 \times$; scale bar, $100 \mu \mathrm{m}$. Data are representative of three independent experiments and were analyzed by unpaired $t$ test. Error bars denote S.D. $* P<0.05 ; * * P<0.01 ; * * * P<0.001$. Con (control)

fatty liver disease via affecting lipid metabolism (Feng et al., 2017), alter glucose and lipid metabolism in type 2 diabetes (Ren et al., 2016), facilitate lipid catabolism in HFD-fed mice (Sun and $\mathrm{Qu}, 2019$ ), and protect blood vessels by regulating cholesterol metabolism (Zhang et al., 2017). In the current study, in vitro experiments also revealed that AP had a significant improvement effect on the accumulated lipid droplets in the PA-induced fat accumulation cell model and markedly decreased the levels of SREBP-1c, SREBP-2, FAS, SCD-1, and HMGCOR in PA-induced HepG2 cells. Therefore, we suggested that AP could significantly ameliorate lipid metabolism and lipid accumulation in vitro. Obesity is a chronic disease mainly caused by lipid metabolism disorder, which is caused primarily by the imbalance of energy intake and consumption and also influenced by genes, environment, lifestyle, endocrine abnormalities, inflammation, intestinal flora, etc. (Xu and Xue, 2016; Huang et al., 2019). HFD is one of the leading causes of obesity, and the mechanism of obesity in C57BL/6J mice induced by HFD is similar to that in the human body (Nakamura et al., 2017). In our study, we constructed the HFD-fed model mice, and the results revealed that body weight, visceral fat weight, and blood lipids were markedly elevated in HFD-induced hyperlipidemia model mice, suggesting a successful establishment of the HFD-fed model mice. Besides, we 

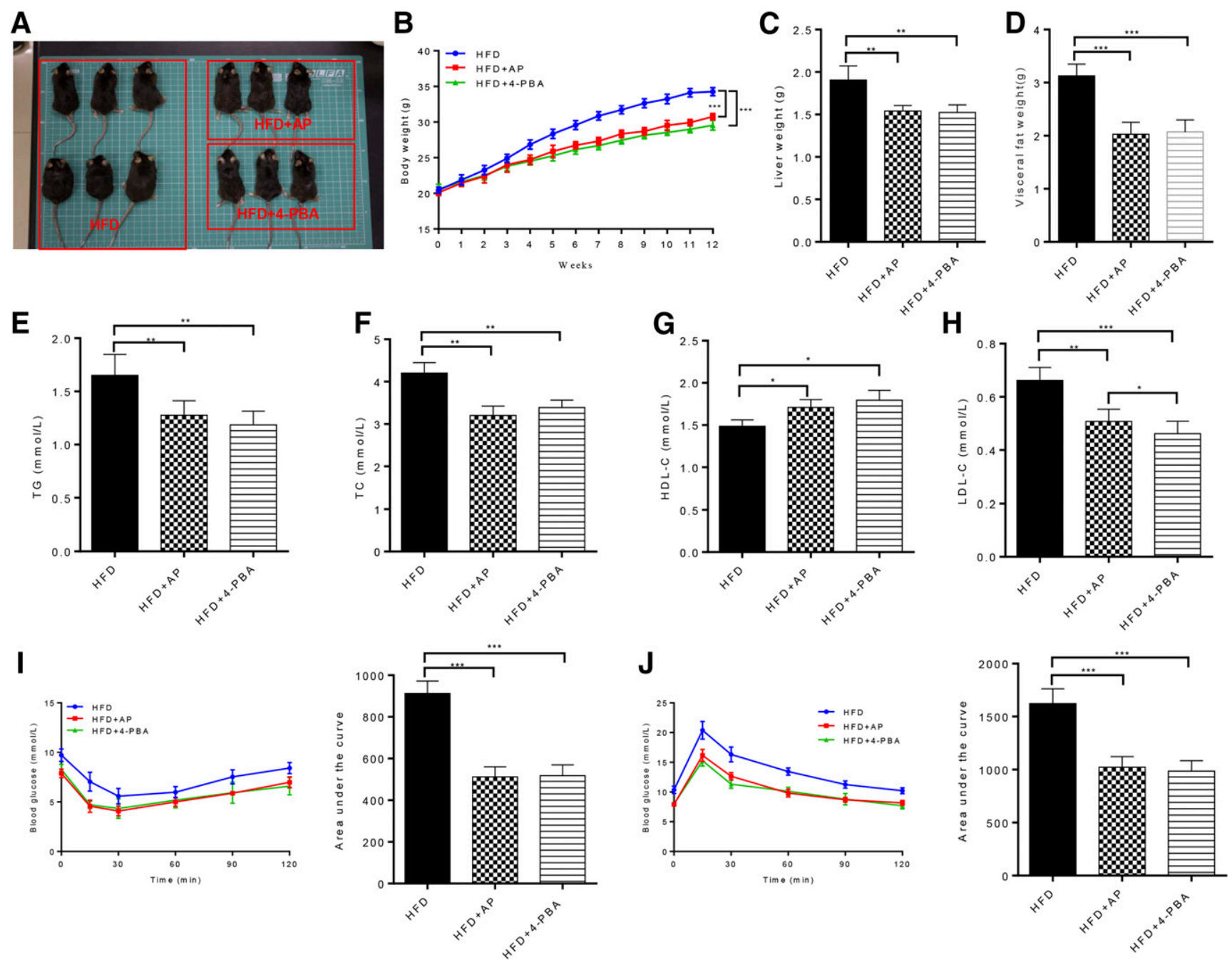

Fig. 6. AP markedly reduced blood lipids and alleviated IR in HFD-fed model mice. (A) HFD was applied to establish the HFD-fed mice model, and AP or 4-PBA was used to treat the mice; six mice were in each group. The body weight (B), liver weight (C), and visceral fat weight (D) were measured at specified points in time. The changes of TG (E), TC (F), HDL-C (G), and LDL-C (H) were also determined using a biochemical analyzer. The concentration of blood glucose was evaluated by IGTT (I) and OGTT (J). Data are representative of three independent experiments and were analyzed by unpaired $t$ test. Error bars denote S.D. $* P<0.05 ; * * P<0.01 ; * * * P<0.001$

further proved that $\mathrm{AP}$ could effectively reduce the body weight, visceral fat weight, lipid accumulation, and blood lipids in HFD-induced hyperlipidemia model mice. Meanwhile, we showed that the food intake of each group remained unchanged for 12 weeks, suggesting that the food intake in each group did not affect the weight of the mice. Previous studies also confirmed that AP could prevent the body weight and visceral obesity of the HFD-induced mice (Sun and $\mathrm{Qu}$, 2019; Su et al., 2020). Besides, previous studies also proved that AP was relevant to gut microbiota (Wang et al., 2019) and inflammation (Li et al., 2017; Kasiri et al., 2018). However, the influence of AP on non-HFD-induced body weight gain remains unclear. In future studies, we will also further explore the influence of AP on non-HFD-induced body weight gain. Moreover, we also testified that AP could effectively reduce SREBP-1c and SREBP-2 expressions in HFD-fed model mice. Therefore, we further established that AP could markedly improve lipid metabolism in vivo.

IR refers to the deficiency of insulin in exerting its normal biologic effects, manifested by the obstacle of glucose utilization in target tissues, resulting in abnormal glucose tolerance (Petersen and Shulman, 2018). Research proved that IR is a vital feature in the development of type 2 diabetes and a key link of metabolic syndrome, which is closely related to obesity, hyperlipidemia, and cardiovascular diseases (Roden et al., 2017). In this study, we revealed that AP could effectively relieve the IR of HFD-induced hyperlipidemia model mice, which might be associated with reducing SREBP-1c and SREBP-2 expression, ERS, and lipid accumulation in HFDfed model mice. In addition to HFD-inducted obesity, other factors can also cause IR, such as oxidative stress, functional defects of related molecules and organelles in cells, deficiency of trace elements, etc. A previous research found that high fructose-induced IR was related to vitamin E contents (Kitagawa et al., 2020). However, the effect of AP on non-HFDinduced IR has not been exactly elucidated. This part of limitation will also be one of our future research directions.

At present, ERS has been proved to be involved in lipid metabolism by a large number of studies (Su et al., 2018; Sun et al., 2018; Zhu et al., 2019). During ERS, activation of 
a series of signaling pathways from the ER to the nucleus is collectively known as the unfolded protein response. This response consists of GRP78 and three receptor proteins (PERK, ATF6, and IRE1) (Zhou et al., 2018; Jia et al., 2019). Among them, p-PERK, p-eIF2 $\alpha$, and CHOP are the main signaling pathways of ERS, and GRP78 is the major marker of ERS (Xu et al., 2017). At the early stage of ERS, GRP7 is separated from PERK, and PERK is dimerized and phosphorylated, resulting in phosphorylation of downstream eIF2 $\alpha$, which then alleviates ERS (Liu et al., 2016). As an ERSspecific transcription factor, CHOP has also been demonstrated to be associated with a variety of cell functional activities, such as proliferation, differentiation, and apoptosis (Zhao et al., 2018). ATF6 is a type II transmembrane protein (Hillary and FitzGerald, 2018), IRE1 is a type of transmembrane protein in ER membrane (Lin et al., 2007). ATF6 and IRE1 is mainly responsible for protein folding and degradation (Sano and Reed, 2013). In this study, the results revealed that AP could significantly downregulate p-PERK, GRP78, p-eIF2 $\alpha$, and $\mathrm{CHOP}$ in PA-induced HepG2 cells, whereas AP did not affect ATF6 and IRE1 expressions in HFD-fed model mice. Therefore, we demonstrated that AP could suppress ERS by regulating p-PERK, GRP78, p-eIF2 $\alpha$, and CHOP in PA-induced HepG2 cells. Moreover, we further revealed that AP, like ERS alleviator (4-PBA), had significant suppressive effects on blood lipids and IR in HFD-fed model mice.

\section{Conclusion}

In summary, we demonstrated that $25 \mu \mathrm{M}$ AP could alleviate diet-induced obesity and obesity-related insulin resistance. Meanwhile, we discovered that AP could reduce lipid deposition. More importantly, we proved that AP could reduce lipid accumulation and insulin resistance by regulating ERS. Therefore, AP has significant contributions to lipid metabolism and insulin resistance, which might provide a basis for the treatment of hyperlipidemia and other related diseases.

\section{Acknowledgments}

We thank the members of Medical Function Experimental Center, North Sichuan Medical University, for enabling us to use their instruments for animal anesthesia and sample analysis.

\section{Authorship Contributions}

Participated in research design: $\mathrm{Wu}, \mathrm{Guo}, \mathrm{Liu}, \mathrm{Yu}$.

Conducted experiments: Wu, Guo, Deng.

Performed data analysis: Wu, Deng, Yu.

Wrote or contributed to the writing of the manuscript: $\mathrm{Wu}, \mathrm{Liu}, \mathrm{Yu}$.

\section{References}

Awad AS, Abd Al Haleem EN, El-Bakly WM, and Sherief MA (2016) Thymoquinone alleviates nonalcoholic fatty liver disease in rats via suppression of oxidative stress, inflammation, apoptosis. Naunyn Schmiedebergs Arch Pharmacol 389: $381-391$.

Chen J-W, Kong Z-L, Tsai M-L, Lo C-Y, Ho C-T, and Lai C-S (2018) Tetrahydrocurcumin ameliorates free fatty acid-induced hepatic steatosis and improves insulin resistance in HepG2 cells. J Food Drug Anal 26:1075-1085.

Chen L, Teng H, and Cao H (2019) Chlorogenic acid and caffeic acid from Sonchus oleraceus Linn synergistically attenuate insulin resistance and modulate glucose uptake in HepG2 cells. Food Chem Toxicol 127:182-187.

Chilloux J and Dumas M-E (2017) Are gut microbes responsible for post-dieting weight rebound? Cell Metab 25:6-7.

DeBose-Boyd RA and Ye J (2018) SREBPs in lipid metabolism, insulin signaling, and beyond. Trends Biochem Sci 43:358-368.

El Barky AR, El-Said KS, Sadek ME-R, and Mohamed TM (2019) Anti-diabetic activity of Egyptian celery apigenin. Asian J Dairy Food Res 38:341-346.

Feng X, Yu W, Li X, Zhou F, Zhang W, Shen Q, Li J, Zhang C, and Shen P (2017) Apigenin, a modulator of PPAR $\gamma$, attenuates HFD-induced NAFLD by regulating hepatocyte lipid metabolism and oxidative stress via Nrf2 activation. Biochem Pharmacol 136:136-149.

Gatineau Eo and Yiannikouris F (2018) Prorenin receptor regulates lipid metabolism via distinct actions on PPAR $\gamma$ and SREBP-2 in liver of male mice. FASEB $J$ 32(suppl 1):760.12

Gröger M, Dinger J, Kiehntopf M, Peters FT, Rauen U, and Mosig AS (2018) Preservation of cell structure, metabolism, and biotransformation activity of liver-onchip organ models by hypothermic storage. Adv Healthc Mater 7:1700616.

Guo X, Liu J, Cai S, Wang O, and Ji B (2016) Synergistic interactions of apigenin, naringin, quercetin and emodin on inhibition of 3T3-L1 preadipocyte differentiation and pancreas lipase activity. Obes Res Clin Pract 10:327-339.

Hernández-Rodas MC, Valenzuela R, Echeverría F, Rincón-Cervera MA, Espinosa A, Illesca P, Muñoz P, Corbari A, Romero N, Gonzalez-Mañan D, et al. (2017) Supplementation with docosahexaenoic acid and extra virgin olive oil prevents liver steatosis induced by a high-fat diet in mice through PPAR- $\alpha$ and Nrf2 upregulation with concomitant SREBP-1c and NF-kB downregulation. Mol Nutr Food Res 61: 1700479 .

Hillary RF and FitzGerald U (2018) A lifetime of stress: ATF6 in development and homeostasis. J Biomed Sci 25:48.

Huang J, Xiong M, Xiao X, Xu X, and Hong X (2019) fNIRS correlates of the development of inhibitory control in young obese subjects. $J$ Integr Neurosci 18: $253-259$.

Iurlaro R and Muñoz-Pinedo C (2016) Cell death induced by endoplasmic reticulum stress. FEBS J 283:2640-2652.

Jia ZL, Cen J, Wang JB, Zhang F, Xia Q, Wang X, Chen XQ, Wang RC, Hsiao CD, Liu $\mathrm{KC}$, et al. (2019) Mechanism of isoniazid-induced hepatotoxicity in zebrafish larvae: activation of ROS-mediated ERS, apoptosis and the Nrf2 pathway. Chemosphere 227:541-550.

Jung UJ, Cho Y-Y, and Choi M-S (2016) Apigenin ameliorates dyslipidemia, hepatic steatosis and insulin resistance by modulating metabolic and transcriptional profiles in the liver of high-fat diet-induced obese mice. Nutrients 8 : 305

Kasiri N, Rahmati M, Ahmadi L, and Eskandari N (2018) The significant impact of apigenin on different aspects of autoimmune disease. Inflammopharmacology 26: 1359-1373.

Khaleel EF, Abdel-Aleem GA, and Mostafa DG (2018) Resveratrol improves high-fat diet induced fatty liver and insulin resistance by concomitantly inhibiting proteolytic cleavage of sterol regulatory element-binding proteins, free fatty acid oxidation, and intestinal triglyceride absorption. Can J Physiol Pharmacol 96: 145-157.

Kim KY, Park KI, Lee SG, Baek SY, Lee EH, Kim SC, Kim SH, Park SG, Yu SN, Oh TW, et al. (2018) Deoxypodophyllotoxin in Anthriscus sylvestris alleviates fat accumulation in the liver via AMP-activated protein kinase, impeding SREBP-1c signal. Chem Biol Interact 294:151-157.

Kitagawa A, Ohta Y, Ohashi K, Yashiro K, and Fukuzawa K (2020) Effect of high fructose-induced metabolic syndrome on tissue vitamin $\mathrm{E}$ and lipid peroxide levels in rats. J Nutr Sci Vitaminol (Tokyo) 66:200-206.

Lebeaupin C, Vallée D, Hazari Y, Hetz C, Chevet E, and Bailly-Maitre B (2018) Endoplasmic reticulum stress signalling and the pathogenesis of non-alcoholic fatty liver disease. J Hepatol 69:927-947.

Li F, Lang F, Zhang H, Xu L, Wang Y, Zhai C, and Hao E (2017) Apigenin alleviates endotoxin-induced myocardial toxicity by modulating inflammation, oxidative stress, and autophagy. Oxid Med Cell Longev 2017:2302896.

Lin JH, Li H, Yasumura D, Cohen HR, Zhang C, Panning B, Shokat KM, Lavail MM and Walter P (2007) IRE1 signaling affects cell fate during the unfolded protein response. Science 318:944-949.

Liu MQ, Chen Z, and Chen LX (2016) Endoplasmic reticulum stress: a novel mechanism and therapeutic target for cardiovascular diseases. Acta Pharmacol Sin 37: $425-443$.

Madunić J, Madunić IV, Gajski G, Popić J, and Garaj-Vrhovac V (2018) Apigenin: a dietary flavonoid with diverse anticancer properties. Cancer Lett 413:11-22.

Maiers JL and Malhi H (2019) Endoplasmic reticulum stress in metabolic liver diseases and hepatic fibrosis. Semin Liver Dis 39:235-248.

Malik SA, Acharya JD, Mehendale NK, Kamat SS, and Ghaskadbi SS (2019) Pterostilbene reverses palmitic acid mediated insulin resistance in HepG2 cells by reducing oxidative stress and triglyceride accumulation. Free Radic Res 53: $815-827$.

Mo J, Zhou Y, Yang R, Zhang P, He B, Yang J, Li S, Shen Z, and Chen P (2019) Ginsenoside Rg1 ameliorates palmitic acid-induced insulin resistance in HepG2 cells in association with modulating Akt and JNK activity. Pharmacol Rep $\mathbf{7 1}$ 1160-1167.

Nabavi SF, Khan H, D'onofrio G, Šamec D, Shirooie S, Dehpour AR, Argüelles S, Habtemariam S, and Sobarzo-Sanchez E (2018) Apigenin as neuroprotective agent: of mice and men. Pharmacol Res 128:359-365.

Nabavi SM, Habtemariam S, Daglia M, and Nabavi SF (2015) Apigenin and breast cancers: from chemistry to medicine. Anticancer Agents Med Chem 15:728-735.

Nakamura Y, Natsume M, Yasuda A, Ishizaka M, Kawahata K, and Koga J (2017) Fructooligosaccharides suppress high-fat diet-induced fat accumulation in C57BL/ 6J mice. Biofactors 43:145-151.

Ni $\mathrm{X}$ and Wang $\mathrm{H}$ (2016) Silymarin attenuated hepatic steatosis through regulation of lipid metabolism and oxidative stress in a mouse model of nonalcoholic fatty liver disease (NAFLD). Am J Transl Res 8:1073-1081.

Pang Q, Zhao Y, Chen X, Zhao K, Zhai Q, and Tu F (2018) Apigenin protects the brain against ischemia/reperfusion injury via caveolin-1/VEGF in vitro and in vivo. Oxid Med Cell Longev 2018:7017204.

Petersen MC and Shulman GI (2018) Mechanisms of insulin action and insulin resistance. Physiol Rev 98:2133-2223.

Ramos-Lopez O, Riezu-Boj JI, Milagro FI, Moreno-Aliaga MJ, and Martinez JA project MENA (2018) Endoplasmic reticulum stress epigenetics is related to adiposity, dyslipidemia, and insulin resistance. Adipocyte 7:137-142. 
Ren B, Qin W, Wu F, Wang S, Pan C, Wang L, Zeng B, Ma S, and Liang J (2016) Apigenin and naringenin regulate glucose and lipid metabolism, and ameliorate vascular dysfunction in type 2 diabetic rats. Eur $J$ Pharmacol 773:13-23.

Ren K, Jiang T, Zhou H-F, Liang Y, and Zhao G-J (2018) Apigenin retards atherogenesis by promoting ABCA1-mediated cholesterol efflux and suppressing inflammation. Cell Physiol Biochem 47:2170-2184.

Roden M, Petersen K, and Shulman G (2017) Insulin resistance in type 2 diabetes. Textbook of Diabetes. 5th ed. (Holt RI, Cockram C, Flyvbjerg A, and Goldstein BJ eds) pp 174-186, John Wiley \& Sons, New York.

Salehi B, Venditti A, Sharifi-Rad M, Kręgiel D, Sharifi-Rad J, Durazzo A, Lucarini M Santini A, Souto EB, Novellino E, et al. (2019) The therapeutic potential of apigenin. Int J Mol Sci 20:1305.

Sano R and Reed JC (2013) ER stress-induced cell death mechanisms. Biochim Biophys Acta 1833:3460-3470.

Su T, Huang C, Yang C, Jiang T, Su J, Chen M, Fatima S, Gong R, Hu X, Bian Z, et al. (2020) Apigenin inhibits STAT3/CD36 signaling axis and reduces visceral obesity. Pharmacol Res 152:104586.

Su Y, Li H, Xu C, Wang X, Xie J, Qin JG, Chen L, and Li E (2018) Endoplasmic reticulum stress mediates 4,5-dichloro-2-n-octyl-4-isothiazolin-3-one (DCOIT)-induced toxicity and liver lipid metabolism changes in Nile tilapia (Oreochromis niloticus). Environ Pollut 242 (Pt B):1981-1987.

Sun Y, Zhang D, Liu X, Li X, Liu F, Yu Y, Jia S, Zhou Y, and Zhao Y (2018) Endoplasmic reticulum stress affects lipid metabolism in atherosclerosis via CHOP activation and over-expression of miR-33. Cell Physiol Biochem 48:1995-2010.

Sun YS and Qu W (2019) Dietary Apigenin promotes lipid catabolism, thermogenesis, and browning in adipose tissues of HFD-Fed mice. Food Chem Toxicol 133:110780.

Suzuki T, Gao J, Ishigaki Y, Kondo K, Sawada S, Izumi T, Uno K, Kaneko K, Tsukita $\mathrm{S}$, Takahashi K, et al. (2017) ER stress protein CHOP mediates insulin resistance by modulating adipose tissue macrophage polarity. Cell Rep 18:2045-2057.

Vrhovac Madunić I, Madunić J, Antunović M, Paradžik M, Garaj-Vrhovac V, Breljak D, Marijanović I, and Gajski G (2018) Apigenin, a dietary flavonoid, induces apoptosis, DNA damage, and oxidative stress in human breast cancer MCF-7 and MDA MB-231 cells. Naunyn Schmiedebergs Arch Pharmacol 391:537-550.

Wang M, Firrman J, Liu L, and Yam K (2019) A review on flavonoid apigenin: dietary intake, $\mathrm{ADME}$, antimicrobial effects, and interactions with human gut microbiota. BioMed Res Int 2019:7010467.

Xu Q, Chen C, Lin A, and Xie Y (2017) Endoplasmic reticulum stress-mediated membrane expression of CRT/ERp57 induces immunogenic apoptosis in drugresistant endometrial cancer cells. Oncotarget 8:58754-58764.

$\mathrm{Xu} \mathrm{S}$ and Xue Y (2016) Pediatric obesity: causes, symptoms, prevention and treatment. Exp Ther Med 11:15-20.

Zhang K, Song W, Li D, and Jin X (2017) Apigenin in the regulation of cholesterol metabolism and protection of blood vessels. Exp Ther Med 13:1719-1724.

Zhao J, Xiang X, Zhang H, Jiang D, Liang Y, Qing W, Liu L, Zhao Q, and He Z (2018) CHOP induces apoptosis by affecting brain iron metabolism in rats with subarachnoid hemorrhage. Exp Neurol 302:22-33.

Zhao N-Q, Li X-Y, Wang L, Feng Z-L, Li X-F, Wen Y-F, and Han J-X (2017) Palmitate induces fat accumulation by activating C/EBP $\beta$-mediated G0S2 expression in HepG2 cells. World J Gastroenterol 23:7705-7715.

Zhou Y, Liu X, Li W, Sun X, and Xie Z (2018) Endoplasmic reticulum stress contributes to the pathogenesis of stress urinary incontinence in postmenopausal women. J Int Med Res 46:5269-5277.

Zhu Y, Guan Y, Loor JJ, Sha X, Coleman DN, Zhang C, Du X, Shi Z, Li X, Wang Z, et al. (2019) Fatty acid-induced endoplasmic reticulum stress promoted lipid accumulation in calf hepatocytes, and endoplasmic reticulum stress existed in the liver of severe fatty liver cows. J Dairy Sci 102:7359-7370.

Address correspondence to: Dr. Yongxiong Yu, College of Animal Sciences and Technology, Southwest University, Chongqing, China. E-mail: yuyongxiong8@126.com 